\title{
AN UNSYMMETRIC FUBINI THEOREM ${ }^{1}$
}

\author{
R. H. CAMERON AND W. T. MARTIN
}

Because of the great variety of ways in which repeated Stieltjes integrals may occur, the ordinary Fubini theorem for Lebesgue integrals has more than one analogue in Lebesgue-Stieltjes (Radon) integrals. ${ }^{2}$ One of these (the symmetric one) is well known. ${ }^{3}$ However, there is another form which is not symmetric, and its proof does not seem to be in the literature, although it is known to many writers, in special cases at least. ${ }^{4}$ This form does not appear to be immediately derivable from the symmetric form, and since it is of interest in various connections, it seems worthwhile to give an explicit statement and proof. We do not follow the usual procedure of beginning with finite limits and then allowing the limits to become infinite because the passage to infinity seems to present difficulties of the same order as the direct proof of the final result itself. The immediate use of infinite limits is made possible by the fact that the symmetric Fubini theorem has already been proved with infinite limits.

THEOREM. Let $k(x)$ be a function of bounded variation on every finite interval. Let $p(x, u)$ be Borel measurable in $(x, u)$; for almost all $x$ with respect to $k(x)$ let it be of bounded variation in $u$ over every finite $u$-interval. Denote by $V(x, u)$ the variation ${ }^{5} V(x, u)=\int_{0+0}^{u+0}\left|d_{\nu} p(x, v)\right|$. Assume that $\int_{-\infty}^{\infty} V(x, u)|d k(x)|$ exists (is finite) for all $u$. Let $s(u)$ be Borel measurable on $(-\infty, \infty)$. Then the existence (finiteness) of either

$$
\int_{-\infty}^{\infty}|s(u)| d_{u} \int_{-\infty}^{\infty} V(x, u)|d k(x)|
$$

1 Presented to the Society, February 24, 1940.

${ }^{2}$ The integrals which occur in this paper are understood to be Lebesgue-Stieltjes (Radon) integrals. See, for example, Saks, Theory of the Integral, 2d revised edition, Warsaw-Lemberg, 1937, pp. 19 and 67. We note that the familiar principles of monotone and dominated convergence in the Lebesgue theory are also valid in this theory; see pages 28 and 29. In this paper we shall not admit $\pm \infty$ as members of our number system; that is, existence implies finiteness.

${ }^{3}$ Saks, loc. cit., p. 81.

${ }^{4}$ See for instance, N. Wiener and H. R. Pitt, On absolutely convergent FourierStieltjes transforms, Duke Mathematical Journal, vol. 4 (1938), pp. 420-436. The reader will note that a form of this theorem was used in passing from line 13 to line 14 on page 421 .

${ }^{5}$ For negative $u$ we understand that $V(x, u)=-\int_{u+0}^{0+0}\left|d_{v} p(x, v)\right|$. Of course $V$ is strictly a variation only for positive $u$.

${ }^{6}$ Clearly it would be sufficient to require that the integral be finite for $u$ ranging over some sequence having $\pm \infty$ as limit points. 
or

$$
\int_{-\infty}^{\infty}|d k(x)| \int_{-\infty}^{\infty}|s(u)| \cdot\left|d_{u} p(x, u)\right|
$$

implies the existence of the other, their equality, and the existence and equality of the following integrals

$$
\int_{-\infty}^{\infty} s(u) d u \int_{-\infty}^{\infty} p(x, u) d k(x)=\int_{-\infty}^{\infty} d k(x) \int_{-\infty}^{\infty} s(u) d_{u} p(x, u) .
$$

We shall first prove this theorem under the following additional assumptions which we shall later successively remove:

(A) $s(u)$ is a polygonal function (that is, a continuous function whose second derivative exists and is zero except at a finite number of points),

(B) $s(u)$ is bounded and vanishes identically outside a finite interval,

(C) $s(u)$ is non-negative, $k(x)$ is monotonic increasing and $p(x, u)$ is monotonic increasing and right continuous in $u$ and $p(x, 0)=0$.

Clearly the additional hypotheses (B) and (C) imply the existence of (1). Also they imply the existence of (2), as one sees in the following manner. Let $n$ be such that $s(u)=0$ when $|u|>n-1$ and such that $s(u)$ is everywhere less than or equal to $n$. Then since $V(x, u)=p(x, u)$ by (C) we see that

$$
\int_{-\infty}^{\infty} s(u) d_{u} p(x, u) \leqq n \int_{-n}^{n} d_{u} p(x, u)=n[V(x, n)-V(x,-n)] .
$$

Also

$$
\int_{-\infty}^{\infty} V(x, u) d k(x)
$$

exists by hypothesis. Hence

$$
\int_{-\infty}^{\infty} d k(x) \int_{-\infty}^{\infty} s(u) d_{u} p(x, u) \leqq n \int_{-\infty}^{\infty} d k(x)[V(x, n)-V(x,-n)] ;
$$

that is, (2) also exists.

Thus under the additional hypotheses (B) and (C) we need only show the equality of (1) and (2) since obviously the right and left members of (3) are equal to (1) and (2) respectively. In order to show the equality of (1) and (2) under (A), (B) and (C) we integrate by parts and use the fact that $s$ vanishes at $-n$ and at $n$. Thus 


$$
\int_{-n}^{n} s(u) d_{u} \int_{-\infty}^{\infty} p(x, u) d k(x)=-\int_{-n}^{n} d s(u) \int_{-\infty}^{\infty} p(x, u) d k(x)
$$

By the symmetric Fubini theorem, we obtain

$$
\int_{-n}^{n} d s(u) \int_{-\infty}^{\infty} p(x, u) d k(x)=\int_{-\infty}^{\infty} d k(x) \int_{-n}^{n} p(x, u) d s(u) .
$$

Integrating the inner integral of the right member of (7) by parts, we have

$$
\int_{-\infty}^{\infty} d k(x) \int_{-n}^{n} p(x, u) d s(u)=-\int_{-\infty}^{\infty} d k(x) \int_{-n}^{n} s(u) d_{u} p(x, u)
$$

and this with (6) and (7) establishes the equality of (1) and (2) and thus in view of the preceding remarks proves the theorem under the additional assumptions (A), (B) and (C).

Now since every bounded Borel measurable function is a repeated limit of continuous (and even of polygonal) functions ${ }^{7}$ we shall show by the repeated use of the principle of dominated convergence that the theorem still holds when we drop (A) and keep only the additional hypotheses (B) and (C). As we have noted, for this purpose it is again only necessary to prove the equality of (1) and (2). For purposes of induction let us assume that $s^{*}(u)=\lim _{j \rightarrow \infty} s_{j}(u)$ $(-\infty<u<\infty)$ where $s_{1}(u), s_{2}(u), \cdots$ is a sequence of non-negative functions each of which is Borel measurable and vanishes when $|u|>n-1$ and each of which is everywhere less than or equal to $n$. Assuming that the conclusion of the theorem holds for each $s_{j}(u)$ when $p$ and $k$ satisfy (C), we shall show that it also holds for the limit function $s^{*}(u)$. By bounded convergence

$$
\lim _{j \rightarrow \infty} \int_{-n}^{n} s_{j}(u) d_{u} \int_{-\infty}^{\infty} p(x, u) d k(x)=\int_{-n}^{n} s^{*}(u) d_{u} \int_{-\infty}^{\infty} p(x, u) d k(x)
$$

and since (4) holds for each $s_{j}(u)$ it follows by dominated convergence that

$$
\begin{aligned}
\lim _{j \rightarrow \infty} \int_{-\infty}^{\infty} d k(x) \int_{-n}^{n} s_{j}(u) d_{u} p(x, u) & =\int_{-\infty}^{\infty} d k(x) \lim _{j \rightarrow \infty} \int_{-n}^{n} s_{j}(u) d_{u} p(x, u) \\
& =\int_{-\infty}^{\infty} d k(x) \int_{-n}^{n} s^{*}(u) d_{u} p(x, u) .
\end{aligned}
$$

\footnotetext{
7 See, for example, de la Vallée Poussin, Intégrales de Lebesgue (Bor el Monograph), 1916, pp. 36, 37.
} 
Thus if the theorem holds under (B) and (C) for one Baire class it holds under (B) and (C) for the next; and hence the theorem holds under $(\mathrm{B})$ and $(\mathrm{C})$ for all Borel measurable functions.

In order to remove (B) (retaining only $(\mathrm{C})$ ) let

$$
s^{n}(u)=\left\{\begin{array}{c}
s(u) \text { if }|u| \leqq n-1 \text { and } s(u) \leqq n \\
0 \text { otherwise }
\end{array}\right.
$$

Then since $s^{n}(u)$ satisfies (B) and (C),

$$
\int_{-\infty}^{\infty} s^{n}(u) d_{u} \int_{-\infty}^{\infty} p(x, u) d k(x)=\int_{-\infty}^{\infty} d k(x) \int_{-\infty}^{\infty} s^{n}(u) d_{u} p(x, u) .
$$

Since either (1) or (2) exists, both these expressions are bounded (in $n$ ). By monotone convergence

$$
\begin{aligned}
\lim _{n \rightarrow \infty} \int_{-\infty}^{\infty} s^{n}(u) d_{u} \int_{-\infty}^{\infty} p(x, u) d k(x) & =\int_{-\infty}^{\infty} s(u) d_{u} \int_{-\infty}^{\infty} p(x, u) d k(x), \\
\lim _{n \rightarrow \infty} \int_{-\infty}^{\infty} d k(x) \int_{-\infty}^{\infty} s^{n}(u) d_{u} p(x, u) & =\int_{-\infty}^{\infty} d k(x) \lim _{n \rightarrow \infty} \int_{-\infty}^{\infty} s^{n}(u) d_{u} p(x, u) \\
& =\int_{-\infty}^{\infty} d k(x) \int_{-\infty}^{\infty} s(u) d_{u} p(x, u) .
\end{aligned}
$$

Thus the theorem holds under (C).

Finally, we can dispense with (C) and assume merely the original hypotheses of the theorem. To show this let

$$
\begin{array}{rlrl}
s_{i}(u) & =\max \left\{(-1)^{i} s(u), 0\right\}, & i & =0,1, \\
k_{i}(x) & =\frac{1}{2}\left\{\int_{0}^{x+0}|d k(\xi)|+(-1)^{i} k(x+0)\right\}, & i & =0,1, \\
p_{i}(x, u) & =\frac{1}{2}\left\{V(x, u)+(-1)^{i}[p(x, u+0)-p(x, 0+0)]\right\}, & \\
& i=0,1 .
\end{array}
$$

Obviously all these functions are Borel measurable and satisfy (C). We first show that the existence of (1) or (2) for $s, p$ and $k$ implies the existence of (1) or (2) respectively for $s_{i}, p_{j}, k_{l}$. Since we always have

$$
\int_{-\infty}^{\infty}|\phi(x)| d f(x) \geqq \int_{-\infty}^{\infty}|\phi(x)| d g(x)
$$

when $f(x)-g(x)$ is non-decreasing, and 


$$
\int_{-\infty}^{\infty} \phi(x)|d f(x)| \geqq \int_{-\infty}^{\infty} \theta(x)|d f(x)|
$$

when $\phi(x) \geqq \theta(x)$, it follows that

$$
\begin{aligned}
\int_{-\infty}^{\infty}|s(u)| d_{u} \int_{-\infty}^{\infty} V(x, u)|d k(x)| & \geqq \int_{-\infty}^{\infty} s_{i}(u) d_{u} \int_{-\infty}^{\infty} V(x, u)|d k(x)| \\
& \geqq \int_{-\infty}^{\infty} s_{i}(u) d_{u} \int_{-\infty}^{\infty} p_{j}(x, u)|d k(x)| \\
& \geqq \int_{-\infty}^{\infty} s_{i}(u) d_{u} \int_{-\infty}^{\infty} p_{j}(x, u) d k_{l}(x)
\end{aligned}
$$

and

$$
\begin{aligned}
\int_{-\infty}^{\infty}|d k(x)| \int_{-\infty}^{\infty}|s(u)|\left|d_{u} p(x, u)\right| & \geqq \int_{-\infty}^{\infty} d k_{l}(x) \int_{-\infty}^{\infty}|s(u)|\left|d_{u} p(x, u)\right| \\
& \geqq \int_{-\infty}^{\infty} d k_{l}(x) \int_{-\infty}^{\infty} s_{i}(u)\left|d_{u} p(x, u)\right| \\
& \geqq \int_{-\infty}^{\infty} d k_{l}(x) \int_{-\infty}^{\infty} s_{i}(u) d_{u} p_{j}(x, u) .
\end{aligned}
$$

Thus $s_{i}, p_{j}, k_{l}$ satisfy the hypotheses of the theorem, as well as (C), and hence

$$
\begin{aligned}
(-1)^{i+j+l} \int_{-\infty}^{\infty} s_{i}(u) d_{u} \int_{-\infty}^{\infty} p_{j}(x, u) d k_{l}(x) & \\
= & (-1)^{i+j+l} \int_{-\infty}^{\infty} d k_{l}(x) \int_{-\infty}^{\infty} s_{i}(u) d_{u} p_{j}(x, u) .
\end{aligned}
$$

Adding these eight equations yields (3), and hence completes the proof of the theorem.

The Massachusetts Institute of Technology 